Research Article

\title{
Gorenstein-Projective Modules over Upper Triangular Matrix Artin Algebras
}

\author{
Dadi Asefa \\ Department of Mathematics, College of Natural and Computational Sciences, Ambo University, Ambo, Ethiopia \\ Correspondence should be addressed to Dadi Asefa; dadi.asefa@ambou.edu.et
}

Received 29 July 2021; Accepted 15 October 2021; Published 19 November 2021

Academic Editor: Francesca Tartarone

Copyright (C) 2021 Dadi Asefa. This is an open access article distributed under the Creative Commons Attribution License, which permits unrestricted use, distribution, and reproduction in any medium, provided the original work is properly cited.

Gorenstein-projective module is an important research topic in relative homological algebra, representation theory of algebras, triangulated categories, and algebraic geometry (especially in singularity theory). For a given algebra $A$, how to construct all the Gorenstein-projective $A$-modules is a fundamental problem in Gorenstein homological algebra. In this paper, we describe all complete projective resolutions over an upper triangular Artin algebra $\Lambda=\left(\begin{array}{cc}A & { }_{A} M_{B} \\ 0 & B\end{array}\right)$. We also give a necessary and sufficient condition for all finitely generated Gorenstein-projective modules over $\Lambda=\left(\begin{array}{cc}A & { }_{A} M_{B} \\ 0 & B\end{array}\right)$.

\section{Introduction}

Auslander and Bridger [1] generalized finitely generated projective modules to modules of Gorenstein dimension zero over two-sided Noetherian rings. After two decades, Enochs and Jenda [2] generalized it to an arbitrary ring and called it Gorenstein-projective modules. After that, this class of modules got special attention and has been studied by many authors (see, e.g., [3-15]). Given an algebra $A$, it is a fundamental problem to determine all the indecomposable finitely generated Gorenstein-projective $A$-modules, which is usually not easy.

Zhang [14] introduced the notion of compatible bimodules and then determined all the Gorenstein-projective modules over upper triangular matrix Artin algebra $\Lambda=\left(\begin{array}{cc}A & { }_{A} M_{B} \\ 0 & B\end{array}\right)$, in terms of Gorenstein-projective $A$ and $B$-modules. This gives us a strong motivation to study Gorenstein-projective modules over upper triangular matrix Artin algebras.

The main aim of this paper is to describe all the complete projective resolutions and all finitely generated Gorensteinprojective modules over an upper triangular matrix Artin algebra $\Lambda=\left(\begin{array}{cc}A & { }_{A} M_{B} \\ 0 & B\end{array}\right)$, by giving the corresponding sufficient and necessary conditions. Although these conditions ((GP1)-(GP5)) are long, they may be helpful, especially in some special cases.

Throughout the paper, $A-$ Mod denotes the category of left $A$-modules and $A$ - mod denotes the category of finitely generated left $A$-modules for a ring $A$.

\section{Preliminaries}

In this section, we recall some basic definitions and facts that will be used throughout the paper.

Following [16], an $A$-module $M$ is said to be Gorensteinprojective, in $A-\operatorname{Mod}$ (resp., in $A-\operatorname{Mod}$ ), if there is an exact sequence of projective modules in $A-\operatorname{Mod}$ (resp., in $A$ - Mod)

$$
\mathscr{P}^{\bullet}:=\cdots \longrightarrow P^{-1} \longrightarrow P^{0} \stackrel{d^{0}}{\longrightarrow} P^{1} \longrightarrow \cdots,
$$

with $\operatorname{Hom}_{A}\left(P^{\bullet}, Q\right)$ being also exact for any projective module $Q$ in $A-\operatorname{Mod}$ (resp., in $A-\bmod$ ), such that $M \cong \operatorname{Ker} d^{0}$. Such $\mathscr{P}^{\bullet}$ is called a complete projective resolution in $A-\operatorname{Mod}$ (resp., $A-\bmod$ ).

2.1. Modules over Upper Triangular Matrix Algebra. Let ${ }_{A} M_{B}$ be an $A-B$ bimodule and 


$$
\Lambda=\left(\begin{array}{cc}
A & { }_{A} M_{B} \\
0 & B
\end{array}\right)=\left\{\left(\begin{array}{cc}
a & m \\
0 & b
\end{array}\right) \mid a \in A, b \in B, m \in M\right\}
$$

be an Artin algebra. Note that addition of $\Lambda$ is the addition of matrix, and the multiplication is given by

$$
\left(\begin{array}{cc}
a & m \\
0 & b
\end{array}\right) \cdot\left(\begin{array}{cc}
a^{\prime} & m^{\prime} \\
0 & b^{\prime}
\end{array}\right)=\left(\begin{array}{cc}
a a^{\prime} & a m^{\prime}+m b^{\prime} \\
0 & b b^{\prime}
\end{array}\right)
$$

Left modules over $\Lambda$ are described with a triple $\left(\begin{array}{l}X \\ Y\end{array}\right)_{\phi}$, where $X$ is an $A$-module and $Y$ is a $B$-module, and $\phi: M \otimes_{B} Y \longrightarrow X$ is an $A$-map. $\Lambda$-map is described with a pair $\left(\begin{array}{l}f \\ g\end{array}\right):\left(\begin{array}{l}X \\ Y\end{array}\right)_{\phi} \longrightarrow\left(\begin{array}{c}X^{\prime} \\ Y^{\prime}\end{array}\right)_{\phi^{\prime}}$, where $f: X \longrightarrow X$ is an $A$-map and $g: Y \longrightarrow Y^{\prime}$ is a $B$-map, such that the diagram

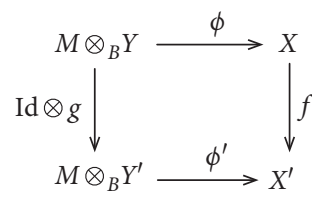

is commutative.

A sequence

$$
0 \longrightarrow\left(\begin{array}{c}
X_{1} \\
Y_{1}
\end{array}\right)_{\phi_{1}} \stackrel{\left(\begin{array}{c}
f_{1} \\
g_{1}
\end{array}\right)}{\longrightarrow}\left(\begin{array}{l}
X_{2} \\
Y_{2}
\end{array}\right)_{\phi_{2}} \stackrel{\left(\begin{array}{c}
f_{2} \\
g_{2}
\end{array}\right)}{\longrightarrow}\left(\begin{array}{c}
X_{3} \\
Y_{3}
\end{array}\right)_{\phi_{3}} \longrightarrow 0
$$

in $\Lambda-\bmod$ is exact if and only if a sequence

$$
0 \longrightarrow X_{1} \stackrel{f_{1}}{\longrightarrow} X_{2} \stackrel{f_{2}}{\longrightarrow} X_{3} \longrightarrow 0
$$

is exact in $A-\bmod$, and a sequence

$$
0 \longrightarrow Y_{1} \stackrel{g_{1}}{\longrightarrow} Y_{2} \stackrel{g_{2}}{\longrightarrow} Y_{3} \longrightarrow 0
$$

is exact in $B-\bmod$.

2.2. Projective Modules. The indecomposable projective (left) $\Lambda$-modules are exactly $\left(\begin{array}{c}P \\ 0\end{array}\right)$ and $\left(\begin{array}{c}M \otimes_{B} Q \\ Q\end{array}\right)$, where $P$ runs over indecomposable projective $A$-modules and $Q$ runs over indecomposable projective $B$-modules.

\section{Complete Projective Resolutions}

The aim of this section is to describe all complete projective resolutions over an upper triangular matrix Artin algebra $\Lambda=\left(\begin{array}{cc}A & { }_{A} M_{B} \\ 0 & B\end{array}\right)$.

3.1. Morphisms of Projective $\Lambda$-Modules. Note that any projective $\Lambda$-module is of the form

$$
X=\left(\begin{array}{c}
P \oplus M \otimes_{B} Q \\
Q
\end{array}\right)
$$

where $P$ and $Q$ are projective $A$-module and projective $B$-module, respectively. For each $i \in \mathbb{Z}$, we write

$$
X^{i}=\left(\begin{array}{c}
P^{i} \oplus M \otimes_{B} Q^{i} \\
Q^{i}
\end{array}\right)
$$
datum

For such a projective $\Lambda$-module $X^{i}$ as above and for any

$$
\left(P^{i}, Q^{i}, \alpha^{i}, \beta^{i}, g^{i}\right)
$$

where $\alpha^{i}: P^{i} \longrightarrow P^{i+1}$ and $\beta^{i}: P^{i} \longrightarrow M \otimes_{B} Q^{i+1}$ are $A$-maps and $g^{i}: Q^{i} \longrightarrow Q^{i+1}$ is $B$-map $\forall i \in \mathbb{Z}$, we put

$$
\begin{aligned}
f^{i}= & \left(\left(\begin{array}{cc}
\alpha^{i} & 0 \\
\beta^{i} & \mathrm{Id}_{M} \otimes g^{i}
\end{array}\right)\right):\left(\begin{array}{c}
P^{i} \oplus M \otimes_{B} Q^{i} \\
g^{i}
\end{array}\right) \\
& \longrightarrow\left(\begin{array}{c}
P^{i+1} \oplus M \otimes_{B} Q^{i+1} \\
Q^{i+1}
\end{array}\right) .
\end{aligned}
$$

Lemma 1. Let $X^{i}$ and $\left(P^{i}, Q^{i}, \alpha^{i}, \beta^{i}, g^{i}\right)$ be given as in (8) and (9). Then, $f^{i}: X^{i} \longrightarrow X^{i+1}$ is a $\Lambda$-map if and only if $f^{i}$ is given in (10).

Proof. First, assume that $f^{i}: X^{i} \longrightarrow X^{i+1}$ is a $\Lambda$-map, where $f^{i}$ is given in (10). Since the diagram

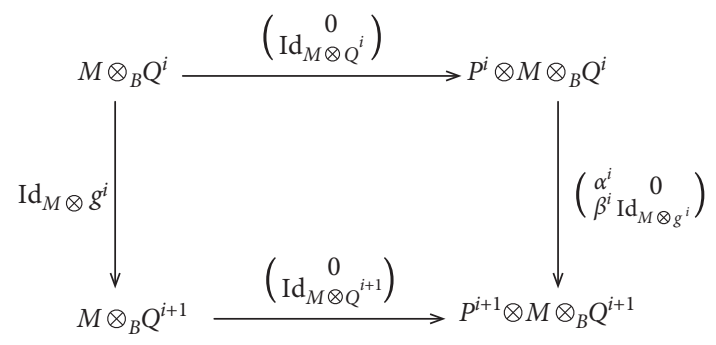

is commutative, we see that $f^{i}: X^{i} \longrightarrow X^{i+1}$ is a $\Lambda$-map, $\forall i \in \mathbb{Z}$.

Conversely, let $f^{i}: X^{i} \longrightarrow X^{i+1}$ be an arbitrary $\Lambda$-map where $X^{i}=\left(\begin{array}{c}P^{i} \oplus M \otimes_{B} Q^{i} \\ Q^{i}\end{array}\right)$. Then, $f^{i}$ is of the form

$$
\left(\left(\begin{array}{cc}
\alpha^{i} & \omega^{i} \\
\beta^{i} & \delta^{i}
\end{array}\right)\right): X^{i} \longrightarrow X^{i+1}
$$

where $\alpha^{i}: P^{i} \longrightarrow P^{i+1}, \quad \beta^{i}: P^{i} \longrightarrow M \otimes_{B} Q^{i+1}, \quad \omega^{i}: M \otimes_{B} Q^{i}$ $\longrightarrow P^{i+1}$, and $\delta^{i}: M \otimes_{B} Q^{i} \longrightarrow M \otimes_{B} Q^{i+1}$ are $A$-maps and $g^{i}: Q^{i} \longrightarrow Q^{i+1}$ is $B$-map, $\forall i \in \mathbb{Z}$. By the definition of morphisms in $\Lambda$-mod, the diagram

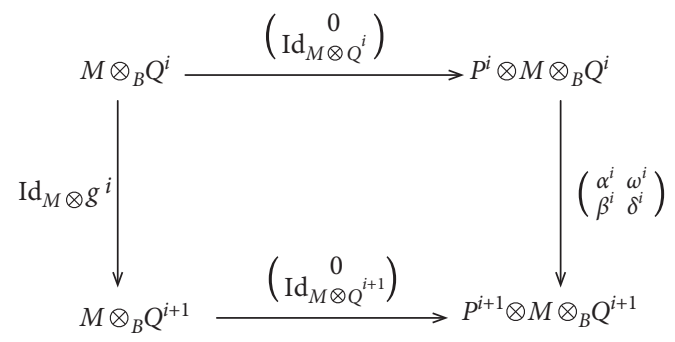

is commutative. This implies that 


$$
\begin{aligned}
& \omega^{i}=0, \\
& \delta^{i}=\operatorname{Id}_{M} \otimes g^{i} \quad \forall i \in \mathbb{Z} .
\end{aligned}
$$

Thus, $f^{i}$ is given as in (10). This completes the proof.

3.2. The Exactness of Morphisms of Projective Modules. Consider the following conditions:

$$
\text { (GP1) } \mathscr{P}^{\bullet}=\cdots \longrightarrow^{\alpha^{-2}} P^{-1} \longrightarrow{ }^{\alpha^{-1}} P^{0} \longrightarrow \alpha^{\alpha^{0}}
$$

$P^{1} \longrightarrow{ }^{\alpha^{1}} \ldots$ is a complex.

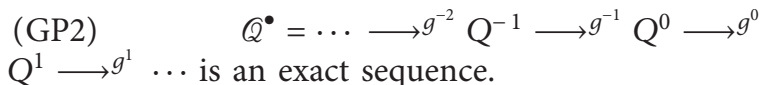

$Q^{1} \longrightarrow g^{1} \ldots$ is an exact sequence.

(GP3) $\beta^{i+1} \alpha^{i}+\left(\operatorname{Id}_{M} \otimes g^{i+1}\right) \beta^{i}=0, \forall i \in \mathbb{Z}$.

(GP4) For any $\left(p^{i+1}, x^{i+1}\right) \in P^{i+1} \oplus M \otimes_{B} Q^{i+1}$ with $\alpha^{i+1}\left(p^{i+1}\right)=0, \quad \beta^{i+1}\left(p^{i+1}\right)+\left(\operatorname{Id}_{N} \otimes g^{i+1}\right)\left(x^{i+1}\right)=0$, there is $\left(p^{\prime i}, x^{\prime i}\right) \in P^{i} \oplus M \otimes_{B} Q^{i}$ such that $p^{i+1}=\alpha^{i}\left(p^{\prime i}\right) \quad$ and $\quad x^{i+1}=\beta^{i}\left(p^{\prime i}\right)+\left(\operatorname{Id}_{M} \otimes g^{i}\right)$ $\left(x^{\prime i}\right), \forall i \in \mathbb{Z}$.

Lemma 2. Let $X^{i}$ be a projective $\Lambda$-module given as in (8) and $f^{i}: X^{i} \longrightarrow X^{i+1}$ be a $\Lambda$-map given as in (10). Then, $\operatorname{Ker} f^{i+1}=\operatorname{Im} f^{i}$ if and only if conditions (GP1)-(GP4) are satisfied.

Proof. By Lemma 1, $f^{i}$ is of the form $f^{i}=$

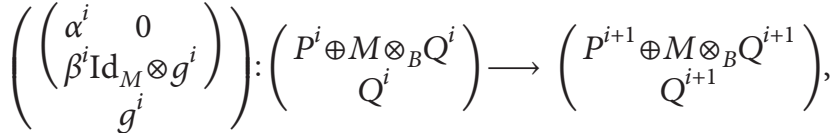

Where $\alpha^{i}: P^{i} \longrightarrow P^{i+1}$ and $\beta^{i}: P^{i} \longrightarrow M \otimes_{B} Q^{i+1}$ and $g^{i}: Q^{i} \longrightarrow Q^{i+1}, \forall i \in \mathbb{Z}$. We see that $\operatorname{Ker} f^{i+1}=$ $\operatorname{Im} f^{i}, \forall i \in \mathbb{Z}$, if and only if

$$
\begin{aligned}
\operatorname{Ker}\left(\begin{array}{cc}
\alpha^{i+1} & 0 \\
\beta^{i+1} & \operatorname{Id}_{M} \otimes g^{i+1}
\end{array}\right) & =\operatorname{Im}\left(\begin{array}{cc}
\alpha^{i} & 0 \\
\beta^{i} & \operatorname{Id}_{M} \otimes g^{i}
\end{array}\right), \\
\operatorname{Ker} g^{i+1} & =\operatorname{Im} g^{i},
\end{aligned}
$$

where

$$
\left(\begin{array}{cc}
\alpha^{i} & 0 \\
\beta^{i} & \operatorname{Id}_{M} \otimes g^{i}
\end{array}\right): P^{i} \oplus M \otimes_{B} Q^{i} \longrightarrow P^{i+1} \oplus M \otimes_{B} Q^{i+1},
$$

$\forall i \in \mathbb{Z}$

Note that $\operatorname{Ker} g^{i+1}=\operatorname{Im} g^{i}, \forall i \in \mathbb{Z}$, if and only if condition (GP2) is satisfied. Thus, it suffices to show that

$$
\operatorname{Ker}\left(\begin{array}{cc}
\alpha^{i+1} & 0 \\
\beta^{i+1} & \operatorname{Id}_{M} \otimes g^{i+1}
\end{array}\right)=\operatorname{Im}\left(\begin{array}{cc}
\alpha^{i} & 0 \\
\beta^{i} & \operatorname{Id}_{M} \otimes g^{i}
\end{array}\right) .
$$

Now, $\operatorname{Im}\left(\begin{array}{cc}\alpha^{i} & 0 \\ \beta^{i} & \operatorname{Id}_{N} \otimes g^{i}\end{array}\right) \subseteq \operatorname{Ker}\left(\begin{array}{cc}\alpha^{i+1} & 0 \\ \beta^{i+1} & \operatorname{Id}_{N} \otimes g^{i+1}\end{array}\right)$ if and only if $\left(\begin{array}{cc}\alpha^{i+1} & 0 \\ \beta^{i+1} & \operatorname{Id}_{N} \otimes g^{i+1}\end{array}\right)\left(\begin{array}{cc}\alpha^{i} & 0 \\ \beta^{i} & \operatorname{Id}_{N} \otimes g^{i}\end{array}\right)=0$, i.e.,

$$
\left(\begin{array}{cc}
\alpha^{i+1} \alpha^{i} & 0 \\
\beta^{i+1} \alpha^{i}+\left(\operatorname{Id}_{N} \otimes g^{i+1}\right) \beta^{i} & \operatorname{Id}_{N} \otimes g^{i+1} g^{i}
\end{array}\right)=0
$$

if and only if conditions (GP1)-(GP3) are satisfied.

$\begin{array}{cc}\text { We } & \text { also see that } \\ \left(\begin{array}{ll}\alpha^{i} & 0\end{array}\right)\end{array}$ $\operatorname{Im}\left(\begin{array}{cc}\alpha^{i} & 0 \\ \beta^{i} & \operatorname{Id}_{N} \otimes g^{i}\end{array}\right), \forall i \in \mathbb{Z}$, if and only if condition (GP4) is satisfied.

Thus, $\operatorname{Ker} f^{i+1}=\operatorname{Im} f^{i}, \forall i \in \mathbb{Z}$, if and only if conditions (GP1)-(GP4) are satisfied.

Consider the following condition (GP5) $\forall i \in \mathbb{Z}$ :

(GP5) For any $c^{i+1} \in \operatorname{Hom}_{A}\left(P^{i+1}, A\right), \quad d^{i+1} \in$ $\operatorname{Hom}_{A}\left(P^{i+1}, M\right), t^{i+1} \in \operatorname{Hom}_{B}\left(Q^{i+1}, B\right)$, such that

$$
\begin{aligned}
c^{i+1} \alpha^{i} & =0, \\
d^{i+1} \alpha^{i}+\left(\operatorname{Id}_{M} \otimes t^{i+1}\right) \beta^{i} & =0, \\
t^{i+1} g^{i} & =0,
\end{aligned}
$$

there are $c^{\prime i+2} \in \operatorname{Hom}_{A}\left(P^{i+2}, A\right), \quad d^{\prime i+2} \in \operatorname{Hom}_{A}$ $\left(P^{i+2}, M\right), t^{i+2} \in \operatorname{Hom}_{B}\left(Q^{i+2}, B\right)$, such that

$$
\begin{aligned}
& c^{i+1}=c^{i+2} \alpha^{i+1}, \\
& d^{i+1}=d^{\prime i+2} \alpha^{i+1}+\left(\operatorname{Id}_{M} \otimes t^{\prime i+2}\right) \beta^{i+1}, \\
& t^{i+1}=t^{\prime i+2} g^{i+1} .
\end{aligned}
$$

Lemma 3. Let $X^{i}$ be a projective $\Lambda$-module given as in (8) and $f: X^{i} \longrightarrow X^{i+1}$ be a $\Lambda$-map given as in (10). Assume that $\operatorname{Ker} f^{i+1}=\operatorname{Im} f^{i}$, i.e., conditions (GP1)-(GP4) are satisfied. Consider the homomorphism of abelian groups $f^{i^{*}}=\operatorname{Hom}_{\Lambda}\left(f^{i}, \Lambda\right): \operatorname{Hom}_{\Lambda}\left(X^{i+1}, \Lambda\right) \longrightarrow \operatorname{Hom}_{\Lambda}\left(X^{i}, \Lambda\right)$. Th en, $\operatorname{Ker} f^{i^{*}}=\operatorname{Im} f^{(i+1)^{*}}, \forall i \in \mathbb{Z}$, if and only if condition (GP5) is satisfied.

Proof. Assume that $\operatorname{Ker} f^{i+1}=\operatorname{Im} f^{i}$. By Lemma 1, we know that $f^{i}: X^{i} \longrightarrow X^{i+1}$ is given as

$$
\begin{aligned}
& \left.f^{i}=\left(\begin{array}{cc}
\alpha^{i} & 0 \\
\beta^{i} & \mathrm{Id}_{M} \otimes g^{i}
\end{array}\right)\right):\left(\begin{array}{c}
P^{i} \oplus M \otimes{ }_{B} Q^{i} \\
g^{i}
\end{array}\right) \\
& \longrightarrow\left(\begin{array}{c}
P^{i+1} \oplus M \otimes{ }_{B} Q^{i+1} \\
Q^{i+1}
\end{array}\right) .
\end{aligned}
$$

Any element in the abelian group $\operatorname{Hom}_{\Lambda}\left(X^{i}, \Lambda\right)=\operatorname{Hom}_{\Lambda}\left(\left(\begin{array}{c}P^{i} \oplus M \otimes_{B} Q^{i} \\ Q^{i}\end{array}\right), \quad\left(\begin{array}{c}A \oplus M \\ B\end{array}\right)\right)$, $\forall i \in \mathbb{Z}$, will be written as 
$h^{i}=\left(\left(\begin{array}{cc}c^{i} & e^{i} \\ d^{i} & s^{i}\end{array}\right)\right):\left(\begin{array}{c}P^{i} \oplus M \otimes_{B} Q^{i} \\ t^{i}\end{array}\right) \longrightarrow\left(\begin{array}{c}A \oplus M \\ B\end{array}\right)$.

(20)

By definition of morphisms in $\Lambda$-mod, the diagram

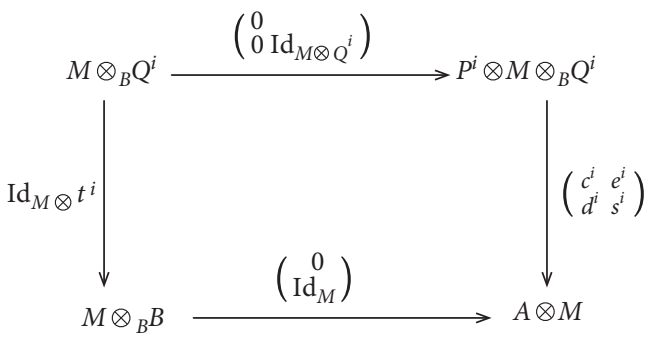

$$
\begin{aligned}
e^{i} & =0, \\
s^{i}=\operatorname{Id}_{M} \otimes t^{i} \quad \forall i & \in \mathbb{Z} .
\end{aligned}
$$

Thus,

$h^{i}=\left(\left(\begin{array}{cc}c^{i} & 0 \\ d^{i} & \operatorname{Id}_{N} \otimes t^{i}\end{array}\right)\right):\left(\begin{array}{c}P^{i} \oplus M \otimes_{B} Q^{i} \\ t^{i}\end{array}\right) \longrightarrow\left(\begin{array}{c}A \oplus M \\ B\end{array}\right)$.

Hence, $\quad f^{i^{*}}=\operatorname{Hom}_{\Lambda}\left(f^{i}, \Lambda\right): \quad \operatorname{Hom}_{\Lambda}\left(X^{i+1}, \Lambda\right) \longrightarrow$ $\operatorname{Hom}_{\Lambda}\left(X^{i}, \Lambda\right), \forall i \in \mathbb{Z}$, is written as

is commutative. This implies that

$$
\begin{aligned}
& f^{i^{*}}=\operatorname{Hom}_{\Lambda}\left(\left(\left(\begin{array}{cc}
\alpha^{i} & 0 \\
\beta^{i} & \operatorname{Id}_{M} \otimes g^{i}
\end{array}\right)\right), \Lambda\right):
\end{aligned}
$$

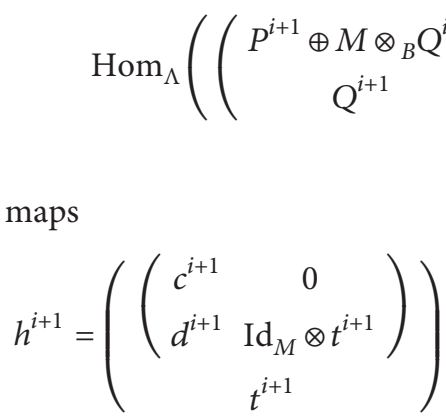

Thus, $f^{i^{*}}$ maps

to

$$
\begin{aligned}
f^{i^{*}}\left(h^{i+1}\right) & =h^{i+1} f^{i}=\left(\left(\begin{array}{cc}
c^{i+1} & 0 \\
d^{i+1} & \operatorname{Id}_{M} \otimes t^{i+1}
\end{array}\right)\left(\begin{array}{cc}
\alpha^{i} & 0 \\
\beta^{i} & \operatorname{Id}_{M} \otimes g^{i}
\end{array}\right)\right) \\
& \left.=\left(\begin{array}{cc}
t^{i+1} g^{i} & \\
c^{i+1} & 0 \\
d^{i+1} \alpha^{i}+\left(\operatorname{Id}_{M} \otimes t^{i+1}\right) \beta^{i} & \operatorname{Id}_{M} \otimes t^{i+1} g^{i}
\end{array}\right)\right) .
\end{aligned}
$$

This means that $h^{i+1}=\left(\left(\begin{array}{cc}c^{i+1} & 0 \\ d^{i+1} & \operatorname{Id}_{M} \otimes t^{i+1}\end{array}\right)\right) \in \operatorname{Ker} f^{i^{*}}$

$t^{i+1}$

$$
c^{i+1} \alpha^{i}=0,
$$$$
d^{i+1} \alpha^{i}+\left(\operatorname{Id}_{M} \otimes t^{i+1}\right) \beta^{i}=0 \text {, }
$$$$
t^{i+1} g^{i}=0 .
$$

$$
\longrightarrow \operatorname{Hom}_{\Lambda}\left(\left(\begin{array}{c}
P^{i} \oplus M \otimes_{B} Q^{i} \\
Q^{i}
\end{array}\right),\left(\begin{array}{c}
A \oplus M \\
B
\end{array}\right)\right)
$$

We see that $h^{i+1}=\left(\left(\begin{array}{cc}c^{i+1} & 0 \\ d^{i+1} & \operatorname{Id}_{M} \otimes t^{i+1}\end{array}\right)\right) \in \operatorname{Im} f^{i+1^{*}}$ if and only if there is $h^{i+2}=\left(\left(\begin{array}{cc}c^{i+2} & 0 \\ d^{i+2} & \operatorname{Id}_{M} \otimes t^{i+2}\end{array}\right)\right)$ such

that

$$
\begin{aligned}
& \left.\left(\begin{array}{cc}
c^{\prime i+2} & 0 \\
d^{\prime i+2} & \operatorname{Id}_{M} \otimes t^{\prime i+2}
\end{array}\right)\left(\begin{array}{cc}
\alpha^{i+1} & 0 \\
\beta^{i+1} & \operatorname{Id}_{M} \otimes g^{i+1}
\end{array}\right)\right) \\
& \left.=\left(\begin{array}{cc}
c^{i+1} & 0 \\
d^{i+1} & \operatorname{Id}_{M} \otimes t^{i+1}
\end{array}\right)\right),
\end{aligned}
$$

which means that

$$
\begin{aligned}
& c^{i+1}=c^{i+2} \alpha^{i+1}, \\
& d^{i+1}=d^{i+2} \alpha^{i+1}+\left(\operatorname{Id}_{M} \otimes t^{i+2}\right) \beta^{i+1}, \\
& t^{i+1}=t^{i+2} g^{i+1} .
\end{aligned}
$$

By conditions (G1)-(G4), we have 


$$
\begin{aligned}
f^{i+1} f^{i} & =\left(\left(\begin{array}{cc}
\alpha^{i+1} & 0 \\
\beta^{i+1} & \mathrm{Id}_{M} \otimes g^{i+1}
\end{array}\right)\left(\begin{array}{cc}
\alpha^{i} & 0 \\
\beta^{i} & \operatorname{Id}_{M} \otimes g^{i}
\end{array}\right)\right) \\
g^{i+1} g^{i} & \\
& =\left(\left(\begin{array}{cc}
\alpha^{i+1} \alpha^{i} & 0 \\
\beta^{i+1} \alpha^{i}+\left(\operatorname{Id}_{N} \otimes g^{i+1}\right) \beta^{i} & \operatorname{Id}_{M} \otimes g^{i+1} g^{i}
\end{array}\right)\right)=0 . \\
g^{i+1} g^{i} &
\end{aligned}
$$

Thus, $\quad f^{i^{*}} f^{i+1^{*}}\left(h^{i+2}\right)=h^{i+2} f^{i+1} f^{i}=0$, i.e., $\quad \operatorname{Im} f^{i+1^{*}} \subseteq$ $\operatorname{Ker} f^{i^{*}}$. Therefore, $\operatorname{Im} f^{i+1^{*}}=\operatorname{Ker} f^{i^{*}}$ if and only if $\operatorname{Im} f^{i+1^{*}} \supseteq \operatorname{Ker} f^{i^{*}}$, if and only if for any $\left.h^{i}=\left(\begin{array}{cc}c^{i+1} & 0 \\ d^{i+1} & \mathrm{Id}_{N} \otimes t^{i+1} \\ & t^{i+1}\end{array}\right)\right): \quad\left(\begin{array}{c}P^{i+1} \oplus M \otimes_{B} Q^{i+1} \\ Q^{i+1}\end{array}\right) \longrightarrow$ $\left(\begin{array}{c}A \oplus M \\ B\end{array}\right)$ with

$$
\begin{aligned}
c^{i+1} \alpha^{i} & =0, \\
d^{i+1} \alpha^{i}+\left(\operatorname{Id}_{M} \otimes t^{i+1}\right) \beta^{i} & =0, \\
t^{i+1} g^{i} & =0,
\end{aligned}
$$

there is

$$
\begin{aligned}
h^{\prime i+2}= & \left.\left(\begin{array}{cc}
c^{i+2} & 0 \\
d^{\prime i+2} & I d_{M} \otimes t^{i+2} \\
t^{i+2}
\end{array}\right)\right):\left(\begin{array}{c}
P^{i+2} \oplus M \otimes_{B} Q^{i+2} \\
Q^{i+2}
\end{array}\right) \\
& \longrightarrow\left(\begin{array}{c}
A \oplus M \\
B
\end{array}\right),
\end{aligned}
$$

such that

$$
\begin{aligned}
c^{i+1} & =c^{i+2} \alpha^{i+1}, \\
d^{i+1} & =d^{i+2} \alpha^{i+1}+\left(\operatorname{Id}_{M} \otimes t^{i+2}\right) \beta^{i+1}, \\
t^{i+1} & =t^{i+2} g^{i+1} .
\end{aligned}
$$

So, by the above arguments, we see that $\operatorname{Im} f^{i+1^{*}}=\operatorname{Ker} f^{i^{*}}, \forall i \in \mathbb{Z}$, if and only if condition (GP5) is satisfied. This completes the proof.

3.3. Main Result. Now, by Lemmas 2 and 3, we get the following sufficient and necessary conditions of any complete projective resolutions over $\Lambda=\left(\begin{array}{cc}A & { }_{A} M_{B} \\ 0 & B\end{array}\right)$.

Theorem 1. Let $X^{i}$ be a projective $\Lambda$-module, given as in (8), and $f^{i}: X^{i} \longrightarrow X^{i+1}$ be a $\Lambda$-map, given as in (10). Then,

$$
x^{\bullet}=\cdots \stackrel{f^{-2}}{\longrightarrow} X^{-1} \stackrel{f^{-1}}{\longrightarrow} X^{0} \stackrel{f^{0}}{\longrightarrow} X^{1} \stackrel{f^{1}}{\longrightarrow} \cdots
$$

is a complete $\Lambda$-projective resolution if and only if conditions (GP1)-(GP5) are satisfied.
3.4. Special Complete Projective Resolutions. We consider the special case of $f: X^{i} \longrightarrow X^{i+1}$ where

$$
\begin{aligned}
f^{i}= & \left(\left(\begin{array}{cc}
\alpha^{i} & 0 \\
\beta^{i} & \operatorname{Id}_{M} \otimes g^{i}
\end{array}\right)\right):\left(\begin{array}{c}
P^{i} \oplus M \otimes_{B} Q^{i} \\
g^{i}
\end{array}\right) \\
& \longrightarrow\left(\begin{array}{c}
P^{i+1} \oplus M \otimes_{B} Q^{i+1} \\
Q^{i+1}
\end{array}\right),
\end{aligned}
$$

and we take $\beta^{i}=0 \forall i \in \mathbb{Z}$, i.e.,

$$
\begin{aligned}
f^{i}= & \left(\left(\begin{array}{cc}
\alpha^{i} & 0 \\
0 & \operatorname{Id}_{M} \otimes g^{i}
\end{array}\right)\right):\left(\begin{array}{c}
P^{i} \oplus M \otimes_{B} Q^{i} \\
g^{i}
\end{array}\right) \\
& \longrightarrow\left(\begin{array}{c}
P^{i+1} \oplus M \otimes_{B} Q^{i+1} \\
Q^{i+1}
\end{array}\right) .
\end{aligned}
$$

Then the condition (GP3) holds for each $i \in Z$. We then get the following special kind of complete projective resolutions over $\Lambda=\left(A_{A} M_{B} 0 B\right)$.

Proposition 1. Let $X^{i}$ be a projective $\Lambda$-module, given as in (8), and $f^{i}: X^{i} \longrightarrow X^{i+1}$ be a $\Lambda$-map, given as in (35). Then,

$$
x^{\bullet}=\cdots \stackrel{f^{-2}}{\longrightarrow} X^{-1} \stackrel{f^{-1}}{\longrightarrow} X^{0} \stackrel{f^{0}}{\longrightarrow} X^{1} \stackrel{f^{1}}{\longrightarrow} \cdots
$$

is a complete $\Lambda$-projective resolution if and only if

$$
\mathscr{P}^{\bullet}=\cdots \stackrel{\alpha^{-2}}{\longrightarrow} P^{-1} \stackrel{\alpha^{-1}}{\longrightarrow} P^{0} \stackrel{\alpha^{0}}{\longrightarrow} P^{1} \stackrel{\alpha^{1}}{\longrightarrow} \cdots
$$

is a complete A-projective resolution,

$$
\mathbb{Q}^{\bullet}=\cdots \stackrel{g^{-2}}{\longrightarrow} Q^{-1} \stackrel{g^{-1}}{\longrightarrow} Q^{0} \stackrel{g^{0}}{\longrightarrow} Q^{1} \stackrel{g^{1}}{\longrightarrow} \cdots
$$

is a complete B-projective resolution, and $M \otimes_{B} Q^{\bullet}$ and $\operatorname{Hom}_{A}\left(\mathscr{P}^{\bullet}, M\right)$ are exact sequences.

Proof. Assume that (36) is a complete $\Lambda$-projective resolution. Then, by Theorem 1, we know that conditions (GP1), (GP2), (GP4), and (GP5) are satisfied.

Let $p^{i+1} \in \operatorname{Ker} \alpha^{i+1}$. Since $\beta^{i+1}=0$, it follows from (GP4) (by taking $x^{i+1}=0$ ) that $p^{i+1} \in \operatorname{Im} \alpha^{i}$, i.e., $\operatorname{Ker} \alpha^{i+1} \subseteq \operatorname{Im} \alpha^{i}$. Together with (GP1), we see that the sequence $\mathscr{P}^{\bullet}$ in (37) is exact.

Since $\beta^{i}=0,(\mathrm{GP} 4)$ means that $M \otimes_{B} Q^{\bullet}$ is an exact sequence.

Since $\beta^{i}=0, \quad(\mathrm{GP} 5)$ means that $\operatorname{Hom}_{A}\left(\mathscr{P}^{\bullet}, A\right)$, $\operatorname{Hom}_{A}\left(\mathscr{P}^{\bullet}, M\right)$, and $\operatorname{Hom}_{B}\left(\mathscr{Q}^{\bullet}, B\right)$ are exact sequences.

Since $\mathscr{P}^{\bullet}$ and $\operatorname{Hom}_{A}\left(\mathscr{P}^{\bullet}, A\right)$ are exact sequences, it follows that $\mathscr{P}^{\bullet}$ is a complete $A$-projective resolution. Similarly, $\mathbb{Q}^{\bullet}$ is a complete $B$-projective resolution.

Conversely, assume that $\mathscr{P}^{\bullet}$ is a complete $A$-projective resolution, $\mathbb{Q}^{\bullet}$ is a complete $B$-projective resolution, and $M \otimes{ }_{B} Q^{\bullet}$ and $\operatorname{Hom}_{A}\left(\mathscr{P}^{\bullet}, M\right)$ are exact sequences. Then, conditions (GP1), (GP2), (GP4), and (GP5) are satisfied, 
where $\beta^{i}=0, \forall i \in \mathbb{Z}$. It follows from Theorem 1 that (36) is a complete $\Lambda$-projective resolution.

\section{Gorenstein-Projective Modules}

In this section, we describe all the Gorenstein-projective modules over $\Lambda=\left(\begin{array}{cc}A & { }_{A} M_{B} \\ 0 & B\end{array}\right)$.

4.1. Main Result. Keeping the notations in Section 3, we put

$$
{ }^{\perp} N=\left\{L \in A-\bmod \mid \operatorname{Ext}_{A}^{i}(L, N)=0, \quad \forall i \geq 1\right\} .
$$

In the next result, we give sufficient and necessary conditions for all Gorenstein-projective modules over $\Lambda=\left(\begin{array}{cc}A & { }_{A} M_{B} \\ 0 & B\end{array}\right)$

Theorem 2. Let $\Lambda=\left(\begin{array}{cc}A & { }_{A} M_{B} \\ 0 & B\end{array}\right)$ be an Artin algebra and $N$ be an $\Lambda$-module. Then, $N$ is a Gorenstein-projective $\Lambda$-module if and only if one of the following cases holds:
(1) $N=\left(\begin{array}{l}L \\ 0\end{array}\right)$ with $L \in G \operatorname{proj} A$ and the sequence $\operatorname{Hom}_{A}\left(\mathscr{P}^{\bullet}, M\right)$ is exact, where $L$ admits a complete A-projective resolution

$$
\ldots \stackrel{\alpha^{-2}}{\longrightarrow} P^{-1} \stackrel{\alpha^{-1}}{\longrightarrow} P^{0} \stackrel{\alpha^{0}}{\longrightarrow} P^{1} \stackrel{\alpha^{1}}{\longrightarrow} \cdots .
$$

(2) $N=\left(\begin{array}{c}M \otimes{ }_{B} K \\ K\end{array}\right)$ with $K \in G \operatorname{proj} B$, where $K$ admits a complete B-projective resolution

$$
\cdots \stackrel{g^{-2}}{\longrightarrow} Q^{-1} \stackrel{g^{-1}}{\longrightarrow} Q^{0} \stackrel{g^{0}}{\longrightarrow} Q^{1} \stackrel{g^{1}}{\longrightarrow} \cdots
$$

such that $\operatorname{Ker}\left(\operatorname{Id}_{M} \otimes g^{i}\right)=M \otimes_{B} \operatorname{Kerg}^{i} \forall i \in \mathbb{Z}$.

(3) $N$ is the $\Lambda$-module:

$$
N=\operatorname{Ker} f^{0}=\left(\begin{array}{c}
\left\{\left(p^{0}, x^{0}\right) \in P^{0} \oplus M \otimes_{B} Q^{0} \mid \alpha^{0}\left(p^{0}\right)=0, \beta^{0}\left(p^{0}\right)+\left(\operatorname{Id}_{M} \otimes g^{0}\right)\left(x^{0}\right)=0\right\} \\
\operatorname{Ker} g^{0}
\end{array}\right),
$$

where $\left(P^{i}, Q^{i}, \alpha^{i}, g^{i}, \beta^{i}\right)$ is as in (9) with $P^{i} \neq 0 \neq Q^{i}$ for some $i$ and $f^{i}$ is given as in (10), such that conditions (GP1)-(GP5) are satisfied.

Proof. First, we prove the sufficiency.
Suppose that case (1) holds. So, $N=\left(\begin{array}{l}L \\ 0\end{array}\right)$ with $L \in G \operatorname{proj} A$ with $L \in G \operatorname{proj} A$, where $L=\operatorname{Ker} \alpha^{0} \in G \operatorname{proj} A$ with a complete $A$-projective resolution

$$
\mathscr{P}^{\bullet}=\cdots \stackrel{\alpha^{-2}}{\longrightarrow} P^{-1} \stackrel{\alpha^{-1}}{\longrightarrow} P^{0} \stackrel{\alpha^{0}}{\longrightarrow} P^{1} \stackrel{\alpha^{1}}{\longrightarrow} \cdots,
$$

such that the sequence $\operatorname{Hom}_{A}\left(\mathscr{P}^{\bullet}, M\right)$ is exact.

Thus, the sequence

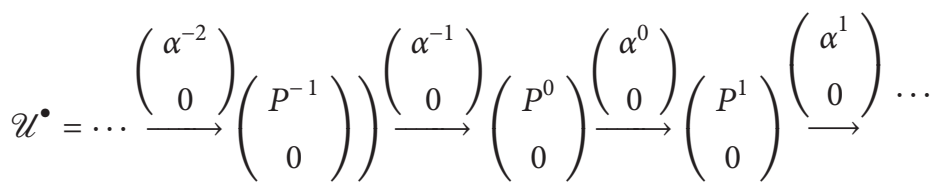

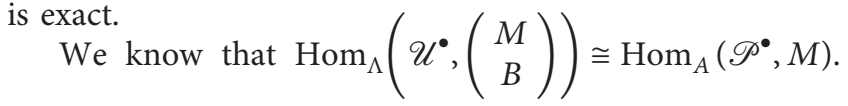

By assumption, the sequence $\operatorname{Hom}_{A}\left(\mathscr{P}^{\bullet}, M\right)$ is exact. Thus, $\operatorname{Hom}_{\Lambda}\left(\mathscr{U}^{\bullet},\left(\begin{array}{c}M \\ B\end{array}\right)\right)$ is exact sequence.

Note that $\operatorname{Hom}_{\Lambda}\left(\mathscr{U}^{\bullet},\left(\begin{array}{c}A \\ 0\end{array}\right)\right) \cong \operatorname{Hom}_{A}(\mathscr{P}, A)$. Since $\mathscr{P}^{\bullet}$ is complete $A$-projective resolution, we have that the sequence $\operatorname{Hom}_{A}\left(\mathscr{P}^{\bullet}, A\right)$ is exact. Thus, $\operatorname{Hom}_{\Lambda}\left(\mathscr{U}^{\bullet},\left(\begin{array}{c}A \\ 0\end{array}\right)\right)$ is exact sequence.
Since $\Lambda=\left(\begin{array}{cc}A & M \\ 0 & B\end{array}\right) \cong\left(\begin{array}{c}A \\ 0\end{array}\right) \oplus\left(\begin{array}{c}M \\ B\end{array}\right)$ as left $\Lambda$-modules, $\operatorname{Hom}_{\Lambda}\left(\mathcal{U}^{\bullet},\left(\begin{array}{cc}A & M \\ 0 & B\end{array}\right)\right)$ is exact. Thus, $\mathcal{U}^{\bullet}$ is a complete $\Lambda$-projective resolution. Thus,

$$
\operatorname{Ker}\left(\begin{array}{c}
\alpha^{0} \\
0
\end{array}\right)=\left(\begin{array}{c}
\operatorname{Ker} \alpha^{0} \\
0
\end{array}\right)=\left(\begin{array}{l}
L \\
0
\end{array}\right)=N
$$

is a Gorenstein-projective $\Lambda$-module. 
Suppose that case (2) holds. That is, $N=\left(\begin{array}{c}M \otimes_{B} K \\ K\end{array}\right)$ with $K \in G$ proj $B$, where $K$ admits a complete $B$-projective resolution

$$
\cdots \stackrel{g^{-2}}{\longrightarrow} Q^{-1} \stackrel{g^{-1}}{\longrightarrow} Q^{0} \stackrel{g^{0}}{\longrightarrow} Q^{1} \stackrel{g^{1}}{\longrightarrow} \cdots
$$

such that $\operatorname{Ker}\left(\operatorname{Id}_{M} \otimes g^{i}\right)=M \otimes{ }_{B} \operatorname{Ker} g^{i} \forall i \in \mathbb{Z}$

Since $\operatorname{Ker}\left(\operatorname{Id}_{M} \otimes g^{i}\right)=M \otimes_{B} \operatorname{Kerg}^{i} \forall i \in \mathbb{Z}$, the sequence

$$
\ldots \longrightarrow \mathrm{Id}_{N} \otimes g^{-2} M \otimes_{B} Q^{-1} \longrightarrow \mathrm{Id}_{M} \otimes g^{-1} M \otimes_{B} Q^{0} \longrightarrow \mathrm{Id}_{M} \otimes g^{0}{ }_{M \otimes_{B} Q^{1} \longrightarrow} \operatorname{Id}_{M} \otimes g^{1} \ldots
$$

is exact. Thus, the sequence

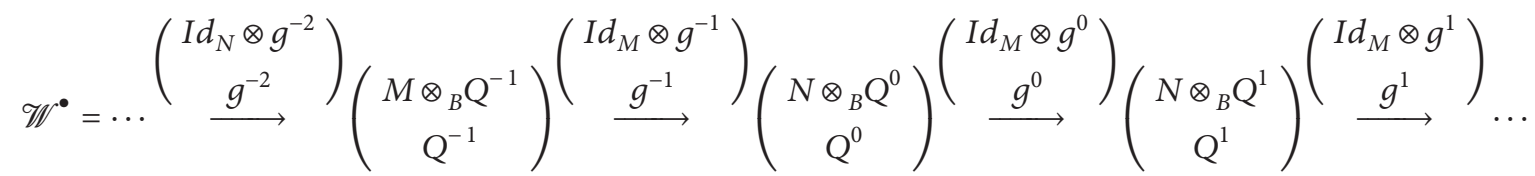

is exact.

Note that $\operatorname{Hom}_{\Lambda}\left(\mathscr{W}^{\bullet},\left(\begin{array}{c}A \\ 0\end{array}\right)\right)=0$. We have that

$$
\operatorname{Hom}_{\Lambda}\left(\mathscr{W}^{\bullet},\left(\begin{array}{c}
M \\
B
\end{array}\right)\right) \cong \operatorname{Hom}_{B}\left(Q^{\bullet}, B\right)
$$

is exact, i.e., the sequence $\operatorname{Hom}_{\Lambda}\left(\mathscr{W}^{\bullet},\left(\begin{array}{c}M \\ B\end{array}\right)\right)$ is exact.

Since $\Lambda=\left(\begin{array}{cc}A & M \\ 0 & B\end{array}\right) \cong\left(\begin{array}{c}A \\ 0\end{array}\right) \oplus\left(\begin{array}{c}M \\ B\end{array}\right)$ as left $\Lambda$-module, we have that $\mathscr{W}^{\bullet}$ is a complete $\Lambda$-projective resolution. Thus,

$$
\operatorname{Ker}\left(\begin{array}{c}
\operatorname{Id}_{M} \otimes g^{0} \\
g^{0}
\end{array}\right)=\left(\begin{array}{c}
\operatorname{Ker}\left(\operatorname{Id}_{M} \otimes g^{0}\right) \\
\operatorname{Ker} g^{0}
\end{array}\right)=\left(\begin{array}{c}
M \otimes_{B} K \\
K
\end{array}\right)=N
$$

is a Gorenstein-projective $\Lambda$-module.

Suppose that case (3) holds. Then, by Theorem 1, the sequence

$$
\mathscr{X}^{\bullet}:=\ldots \stackrel{f^{-2}}{\longrightarrow} X^{-1} \stackrel{f^{-1}}{\longrightarrow} X^{0} \stackrel{f^{0}}{\longrightarrow} X^{1} \stackrel{f^{1}}{\longrightarrow} \cdots
$$

is a complete $\Lambda$-projective resolution, where $X^{i}$ is as given in (8). Thus, $G=\operatorname{Ker} f^{0}$ is a Gorenstein-projective $\Lambda$-module.

Now, we show the necessity. Let $N$ be a Gorensteinprojective $\Lambda$-module. Then, $N=\operatorname{Ker} f^{0}$, where $f^{i}$ is the $\Lambda$-map occurring in a complete $\Lambda$-projective resolution of $x^{\bullet}$. By Theorem $1, X^{\bullet}$ is of form (33), where $X^{i}$ and $f^{i}$ are given in (8) and (10), respectively, satisfying conditions (GP1)-(GP5).

If $Q^{i}=0, \forall i \in \mathbb{Z}$, in (8), then $\beta^{i}=0$ and $g^{i}=0, \forall i \in \mathbb{Z}$. By (GP1) and (GP4), we see that

$$
\mathscr{P}^{\bullet}: \ldots \stackrel{\alpha^{-2}}{\longrightarrow} P^{-1} \stackrel{\alpha^{-1}}{\longrightarrow} P^{0} \stackrel{\alpha^{0}}{\longrightarrow} P^{1} \stackrel{\alpha^{1}}{\longrightarrow} \cdots
$$

is a complete $A$-projective resolution, and (GP5) means that $\operatorname{Hom}_{A}\left(\mathscr{P}^{\bullet}, M\right)$ is exact. Thus, $N=\operatorname{Ker} f^{0}=\left(\begin{array}{c}\operatorname{Ker} \alpha^{0} \\ 0\end{array}\right)$ is of
form $(1)$.

If $P^{i}=0, \forall i \in \mathbb{Z}$, in (8), then $\alpha^{i}=0$ and $\beta^{i}=0, \forall i \in \mathbb{Z}$. By (GP2) and (GP5), we have that

$$
Q^{\bullet}: \cdots \stackrel{g^{-2}}{\longrightarrow} Q^{-1} \stackrel{g^{-1}}{\longrightarrow} Q^{0} \stackrel{g^{0}}{\longrightarrow} Q^{1} \stackrel{g^{1}}{\longrightarrow} \cdots
$$

is a complete $B$-projective resolution, and (GP5) means that $\operatorname{Ker}\left(\operatorname{Id}_{N} \otimes g^{i}\right)=N \otimes \operatorname{Ker} g^{i} \forall i \in \mathbb{Z}$. Thus, $\operatorname{Ker} g^{0} \in G \operatorname{proj} B$, and $N=\operatorname{Ker} f^{0}=\left(\begin{array}{c}N \otimes_{B} \operatorname{Ker} g^{0} \\ \operatorname{Kerg} g^{0}\end{array}\right)$ is of form (2).

If $P^{i} \neq 0$ and $Q^{i} \neq 0$ for some $i \in \mathbb{Z}$ in (9), then $N=\operatorname{Ker} f^{0}$ is of form (3) by Theorem 1. This completes the proof.

\subsection{Special Gorenstein-Projective Modules}

Corollary 1. Suppose that $L$ is a Gorenstein-projective A-module with respect to the complete A-projective resolution

$$
\mathscr{P}^{\bullet}=\cdots \stackrel{\alpha^{-2}}{\longrightarrow} P^{-1} \stackrel{\alpha^{-1}}{\longrightarrow} P^{0} \stackrel{\alpha^{0}}{\longrightarrow} P^{1} \stackrel{\alpha^{1}}{\longrightarrow} \cdots,
$$

and suppose that $K$ is a Gorenstein-projective B-module with respect to the complete $B$-projective resolution

$$
Q^{\bullet}=\cdots \stackrel{g^{-2}}{\longrightarrow} Q^{-1} \stackrel{g^{-1}}{\longrightarrow} Q^{0} \stackrel{g^{0}}{\longrightarrow} Q^{1} \stackrel{g^{1}}{\longrightarrow} \cdots .
$$

Assume that $M \otimes_{B} \widehat{Q}^{\bullet}$ and $\operatorname{Hom}_{A}\left(\mathscr{P}^{\bullet}, M\right)$ are exact sequences. Then, $\left(\begin{array}{c}L \\ o\end{array}\right) \oplus\left(\begin{array}{c}N \otimes_{B} K \\ K\end{array}\right)$ is a Gorenstein-projective $\Lambda$-module with respect to the complete $\Lambda$-projective resolution 


$$
\mathscr{X}^{\bullet}=\cdots \stackrel{f^{-1}}{\longrightarrow}\left(\begin{array}{c}
P^{0} \\
0
\end{array}\right) \oplus\left(\begin{array}{c}
M \otimes Q^{0} \\
Q^{0}
\end{array}\right) \stackrel{f^{0}}{\longrightarrow}\left(\begin{array}{c}
P^{1} \\
0
\end{array}\right) \oplus\left(\begin{array}{c}
M \otimes Q^{1} \\
Q^{1}
\end{array}\right) \stackrel{f^{1}}{\longrightarrow} \cdots
$$

where $\quad \Lambda$-map is $\quad f^{i}=\left(\begin{array}{c}\left(\begin{array}{c}\alpha^{i} \\ 0\end{array}\right) \\ 0 \\ 0\end{array} \quad\left(\begin{array}{c}\operatorname{Id}_{M} \otimes g^{i} \\ g^{i}\end{array}\right)\right)$ :
$\left(\begin{array}{c}P^{i} \\ 0\end{array}\right) \oplus\left(\begin{array}{c}M \otimes_{B} Q^{i} \\ Q^{i}\end{array}\right) \longrightarrow\left(\begin{array}{c}P^{i+1} \\ 0\end{array}\right) \oplus\left(\begin{array}{c}M \otimes_{B} Q^{i+1} \\ Q^{i+1}\end{array}\right)$.

Proof. Since $M \otimes_{B} Q^{\bullet}$ is exact, we have that $\mathscr{X}^{\bullet}$ is exact.

Since each term in the complex $\mathscr{X}^{\bullet}$ is projective, applying $\operatorname{Hom}_{\Lambda}\left(\mathscr{X}^{\bullet},-\right)$ to the exact sequence

$$
0 \longrightarrow\left(\begin{array}{c}
M \\
0
\end{array}\right) \longrightarrow\left(\begin{array}{c}
M \\
B
\end{array}\right) \longrightarrow\left(\begin{array}{l}
0 \\
B
\end{array}\right) \longrightarrow 0
$$

we get following exact sequence of complexes:

$$
\begin{aligned}
0 & \longrightarrow \operatorname{Hom}_{\Lambda}\left(\mathscr{X}^{\bullet},\left(\begin{array}{c}
M \\
0
\end{array}\right)\right) \\
& \longrightarrow \operatorname{Hom}_{\Lambda}\left(\mathscr{X}^{\bullet},\left(\begin{array}{c}
M \\
B
\end{array}\right)\right) \\
& \longrightarrow \operatorname{Hom}_{\Lambda}\left(\mathscr{X}^{\bullet},\left(\begin{array}{c}
0 \\
B
\end{array}\right)\right) \longrightarrow 0 .
\end{aligned}
$$

Since $Q^{\bullet}$ is a complete $B$-projective resolution, it follows that $\operatorname{Hom}_{\Lambda}\left(\mathscr{X}^{\bullet},\left(\begin{array}{c}0 \\ B\end{array}\right)\right) \cong \operatorname{Hom}_{B}\left(Q^{\bullet}, B\right)$ is exact. By assumption, $\operatorname{Hom}_{A}\left(\mathscr{Q}^{\bullet}, M\right)$ is exact. Thus, it follows that $\operatorname{Hom}_{\Lambda}\left(\mathscr{X}^{\bullet},\left(\begin{array}{c}M \\ B\end{array}\right)\right)$ is also exact.

Since $\operatorname{Hom}_{\Lambda}\left(\mathscr{X}^{\bullet},\left(\begin{array}{c}A \\ 0\end{array}\right)\right) \cong \operatorname{Hom}_{A}\left(\mathscr{P}^{\bullet}, A\right) \quad$ and $\operatorname{Hom}_{A}\left(\mathscr{P}^{\bullet}, A\right)$ is exact, it follows that $\operatorname{Hom}_{\Lambda}\left(\mathscr{X}^{\bullet},\left(\begin{array}{c}A \\ 0\end{array}\right)\right)$ is exact.

All together, we see that $\mathscr{X}^{\bullet}$ is a complete $\Lambda$-projective resolution. Thus, $\operatorname{Ker} f^{0}=\left(\begin{array}{c}L \\ 0\end{array}\right) \oplus\left(\begin{array}{c}M \otimes_{B} K \\ K\end{array}\right)$ is a Gorenstein-projective $\Lambda$-module.

\section{Data Availability}

No datasets were generated or analyzed during the current study.

\section{Conflicts of Interest}

The author declares that there are no conflicts of interest.
[2] E. E. Enochs and O. M. G. Jenda, "Gorenstein injective and projective modules," Mathematische Zeitschrift, vol. 220, no. 4, pp. 611-633, 1995.

[3] D. Asefa, "Gorenstein-projective modules over Morita rings," Algebra Colloquium, vol. 28, no. 3, pp. 521-532, 2021.

[4] M. Auslander and I. Reiten, "Applications of contravariantly finite subcategories," Advances in Mathematics, vol. 86, no. 1, pp. 111-152, 1991.

[5] L. L. Avramov and A. Martsinkovsky, "Absolute,relative, and Tate cohomology of modules of finite Gorenstein dimension," Proceedings of the London Mathematical Society, vol. 85, no. 3, pp. 393-440, 2002.

[6] A. Beligiannis, "Cohen-Macaulay modules, (co) torsion pairs and virtually Gorenstein algebras," Journal of Algebra, vol. 288, no. 1, pp. 137-211, 2005.

[7] A. Beligiannis, "On algebras of finite cohen-Macaulay type," Advances in Mathematics, vol. 226, no. 2, pp. 1973-2019, 2011.

[8] D. Happel, "On Gorenstein algebras," Representation Theory of Finite Groups and Finite-Dimensional Algebras, vol. 95, pp. 389-404, 1991.

[9] H. Holm, "Gorenstein homological dimensions," Journal of Pure and Applied Algebra, vol. 189, no. 1-3, pp. 167-193, 2004.

[10] X.-H. Luo and P. Zhang, "Separated monic representations I: Gorenstein-projective modules," Journal of Algebra, vol. 479, pp. 1-34, 2017.

[11] C. M. Ringel and P. Zhang, "Representations of quivers over the algebra of dual numbers," Journal of Algebra, vol. 475, pp. 327-360, 2017.

[12] B. L. Xiong and P. Zhang, "Gorenstein-projective modules over triangular matrix Artin algebras," Journal of Algebra and Its Applications, vol. 11, no. 4, Article ID 1250066, 2012.

[13] P. Zhang, "Monomorphism Categories, cotilting theory, and Gorenstein-projective modules," Journal of Algebra, vol. 339, no. 1, pp. 181-202, 2011.

[14] P. Zhang, "Gorenstein-projective modules and symmetric recollements," Journal of Algebra, vol. 388, pp. 65-80, 2013.

[15] P. Zhang and B.-L. Xiong, "Separated monic representations II: frobenius subcategories and RSS equivalences," Transactions of the American Mathematical Society, vol. 372, no. 2, pp. 981-1021, 2019.

[16] E. E. Enochs and O. M. G. Jenda, "Relative homological algebra," De Gruyter Expositions in Mathematics, vol. 30, 2000.

\section{References}

[1] M. Auslander and M. Bridger, Stable Module Theory, Memoirs of the American Mathematical Society, Providence, RI, USA, 1969. 